\title{
Simultaneous Multi-Bit Recording and Driveless Reading for Permanent Storage in Fused Silica
}

\author{
Manabu SHIOZAWA ${ }^{* 1}$, Takao WATANABE ${ }^{* 1}$, Ryo IMAI ${ }^{* 1}$, Mariko UMEDA ${ }^{* 1}$, Toshiyuki MINE*1, \\ Yasuhiko SHIMOTSUMA ${ }^{* 2}$, Masaaki SAKAKURA ${ }^{* 3}$, Kiyotaka MIURA ${ }^{* 2}$ and Koichi WATANABE ${ }^{* 1}$ \\ ${ }^{* 1}$ Central Research Laboratory, Hitachi, Ltd., 1-280 Higashi-Koigakubo, Kokubunji, Tokyo 185- \\ 601, Japan \\ E-mail: manabu.shiozawa.ar@hitachi.com \\ ${ }^{* 2}$ Department of Material Chemistry, Kyoto University, Kyotodaigaku-Katsura, Nishikyo-ku, Kyoto \\ 615-8510, Japan \\ ${ }^{*}$ Society-Academia Collaboration for Innovation, Kyoto University, Yoshida-Honmachi, Sakyo-ku, \\ Kyoto 605-8501, Japan
}

\begin{abstract}
A storage system using fused silica is a promising way to store historically valuable data permanently. To increase recording capacity, we studied the recording and reading conditions for a multilayer sample. The 26-layer sample, which had a recording capacity equivalent to that of a digital versatile disc (DVD), was made with a femtosecond laser and a spatial light modulator (SLM). The recording pulse energy and interlayer crosstalk were reduced by correcting spherical aberration. The sample was evaluated and read with an optical microscope. The signal-to-noise ratio (SNR) criterion of $15 \mathrm{~dB}$ was satisfied in all 26 layers by using signal processing. Thermal resistance at $1,000{ }^{\circ} \mathrm{C}$ for 120 minutes was demonstrated, suggesting a semi-permanent lifetime.
\end{abstract}

DOI: $10.2961 / \mathrm{jlmn} .2014 .01 .0001$

Keywords: storage, fused silica, femtosecond laser, spatial light modulator, non-linear optical effect, signal-to-noise ratio

\section{Introduction}

In recent years, optical discs and HDDs have been widely used due to their convenience. However, in terms of lifetime, these media are not suitable for storing historically valuable data permanently. The essential requirements for permanent storage are 1) the permanent lifetime of a medium without temperature or humidity control, and 2) a simple readout system that does not depend on specified reading drives.

One candidate for fulfilling these requirements is a system using fused silica, which is thermally and chemically stable, as a recording medium. Several studies have been reported on making microstructures such as dots [1-6], nano-gratings [7-9], and waveguides [10-15] in fused silica with a short-pulse laser and on observations of these microstructures with a microscope. In previous work, we have demonstrated the derivation of recording and reading conditions for a four-layer sample that had a recording capacity equivalent to that of a CD by using evaluation indices $[16,17]$. However, in order to record data of historical paintings and sculptures, the recording capacity must be increased. There are several ways of doing this, such as narrow dot-pitch recording, multi-layer recording and lightphase recording [18]. Considering a simple readout system such as an optical microscope, the multi-layer recording would be the most promising.

We therefore studied the recording and reading conditions for a 26-layer sample with the aim of achieving a capacity comparable with that of a digital versatile disc (DVD). The sample was made using a femtosecond laser and a spatial light modulator (SLM) and was evaluated using the signal-to-noise ratio (SNR) measured with an optical microscope. Spherical aberration was corrected in the recording in order to suppress interlayer crosstalk.

\section{Experimental Method}

The schematic of an optical setup for recording is shown in Fig. 1. In order to create multiple beam spots, a mode-locked, regeneratively amplified Ti:Sapphire laser and an SLM were used. The laser generates laser pulses with a wavelength of $800 \mathrm{~nm}$ and a pulse width of $120 \mathrm{fs}$ at the repetition rate of $1 \mathrm{kHz}$. The SLM modulates a phase of the incident laser beam with a computer-generated hologram displayed on the SLM in accordance with a recording pattern. Lens 1 focuses the laser beam at a knife edge and the knife edge cuts unnecessary light. The objective lens focuses the modulated laser beam inside a fused silica sample and forms a multi-spot. The number of the spots was approximately 100 and dots of the same number were recorded simultaneously. The objective lens has a correction mechanism for spherical aberration that ranges from 0 to $1.2 \mathrm{~mm}$. The numerical aperture of the lens is 0.70 . Exposure time and pulse energy of the laser were controlled with a mechanical shutter and a variable ND filter, respectively. Due to the limitation of response speed of the mechanical shutter, recording speed was approximately 1.5 kbps. The recording speed could be increased by using light modulation device with higher response speed than the shutter.

A commercial fused silica sample was used as the recording medium. Although there is no actual layer in the sample, the laser beam is absorbed only at the focal point 
by non-linear optical effect, resulting in the formation of fine recording dots. By changing the focal point toward a depth direction, 26 recorded layers were made.

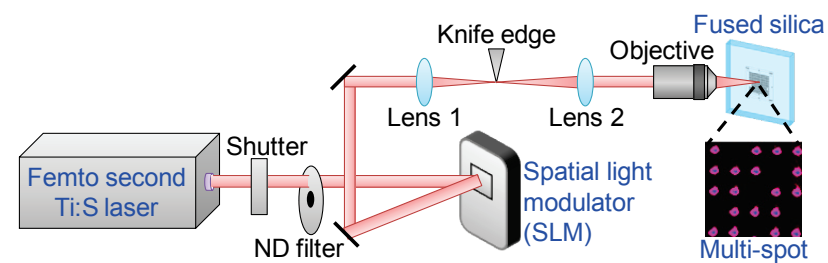

Fig. 1 Schematic of optical setup.

The recorded data were read and evaluated using a commercially available optical microscope with a magnification of 19.5. Correction mechanism for spherical aberration is not equipped with the microscope and it is optimized for observation of surface. An example of the microscopic image of a recorded sample is shown in Fig. 2. Black dots were observed at points where the multi-spot were formed and bright areas called "space" were observed at nonrecorded areas. Digital data can be recorded and read by making these dots and spaces correspond to bit "one" and bit "zero", respectively. The data were decoded on the basis of the brightness of each point of the image and the bit error rate (bER) was calculated by comparing with the binary data of the original data. Figure 3 shows the method we used to evaluate recording quality. We defined the SNR as follows:

$$
\mathrm{SNR}=20 \log \left(V_{\text {Signal }} / \sqrt{{\sigma_{\mathrm{d}}}^{2}+\sigma_{\mathrm{s}}^{2}}\right),
$$

where $V_{\text {Signal }}$ is the difference between the average brightness of the dots and spaces and $\sigma_{\mathrm{d}}$ and $\sigma_{\mathrm{s}}$ are the standard deviations of the brightness of the dots and spaces, respectively. The relationship between the bER and the SNR is shown in Fig. 4. The SNR has a strong correlation with the bER. Assuming that a standard level of error correction code used in optical discs is utilized, we determine the criterion of the SNR to be $15 \mathrm{~dB}$, equivalent to $1 \times 10^{-3}$ of the bit error rate.

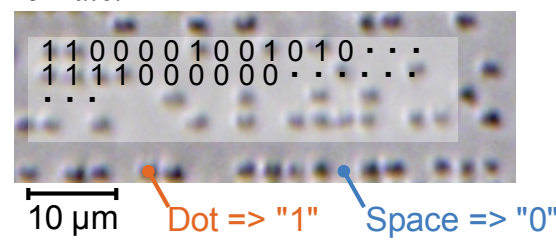

Fig. 2 Example of microscopic image of recorded sample.

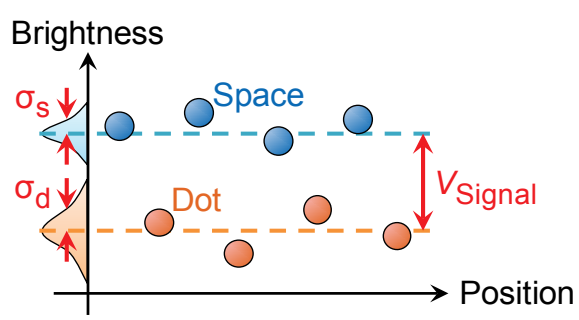

Fig. 3 Evaluation method for recording quality.

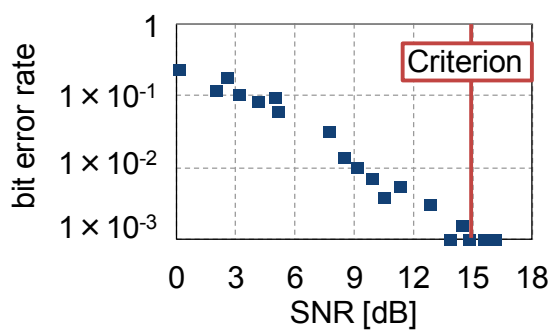

Fig. 4 Relationship between bER and SNR.

\section{Results and Discussion}

\subsection{Derivation of recording condition for 26-layer sample}

To realize a multi-layer sample, there are two challenges regarding the recording condition. One is ensuring recording quality in each layer and the other is reducing interlayer crosstalk. Figure 5 shows the relationship between an SNR and the recording pulse energy in a single layer sample recorded at a depth of $600 \mu \mathrm{m}$ below the surface. The SNR changed depending on the recording pulse energy, indicating that it is necessary to select the optimum energy to obtain the SNR fulfilling the criterion of $15 \mathrm{~dB}$. Figure 5 also shows that the optimum energy was reduced by correcting the spherical aberration, suggesting that the laser energy density inside the fused silica increased by the correction.

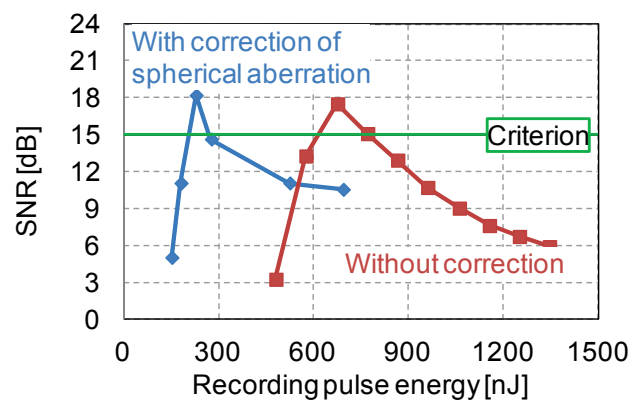

Fig. 5 Relationship between SNR and recording energy.

Figure 6 shows the comparison of a lateral view of a dot recorded at the depth of $600 \mu \mathrm{m}$. The dot length decreased from approximately $40 \mu \mathrm{m}$ to $25 \mu \mathrm{m}$ when the spherical aberration was corrected. This suggests that interlayer-crosstalk can be decreased by correcting the spherical aberration. The method we used for evaluating the interlayer crosstalk is shown in Fig. 7. Although data was recorded only on one layer in the evaluation sample, transmitted dots, which would be interlayer crosstalk, were observed at the other measuring point. We defined the normalized interlayer-crosstalk noise $\left(N_{\mathrm{CTnorm}}\right)$ as follows:

$$
N_{\text {CTnorm }}=\frac{\sigma_{\mathrm{CT}}}{V_{\text {Signal }}},
$$

where $\sigma_{\mathrm{CT}}$ is the interlayer-crosstalk noise, which is the standard deviation of the brightness at the measuring point, and $V_{\text {Signal }}$ is the difference between the average brightness values of the dots and spaces on the recorded layer. The relationship between $N_{\text {CTnorm }}$ and the distance from the recorded layer is plotted in Fig. 8. It decreased with increasing the distance from the recorded layer. Furthermore, the reduction in $N_{\text {CTnorm }}$ by correcting the spherical aberration was confirmed. Figure 9 shows the SNR estimation for the 
26-layer sample. Based on the results of Fig. 8, the noise of the 26-layer sample was calculated as follows:

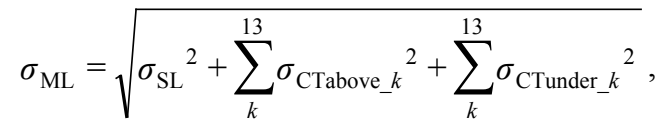

where $\sigma_{\mathrm{SL}}$ is the standard deviation of the brightness of the dots and spaces on the recorded layer and $\sigma_{\mathrm{CTabove}-k}$ and $\sigma_{\text {CTunder } k} k$ are the interlayer-crosstalk noises on the $k$-th measuring point above and under the recorded layer, respectively. The SNR of the 26-layer sample is the ratio of the signal of the recorded layer to $\sigma_{\mathrm{ML}}$. The result of Fig. 9 indicates that the interlayer distance can be decreased by correcting the spherical aberration and the SNR criterion can be satisfied by setting the interlayer distance to more than $40 \mu \mathrm{m}$.

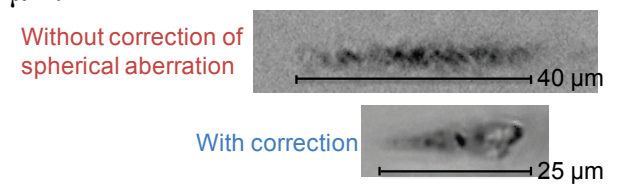

Fig. 6 Comparison of side view of recorded dot.

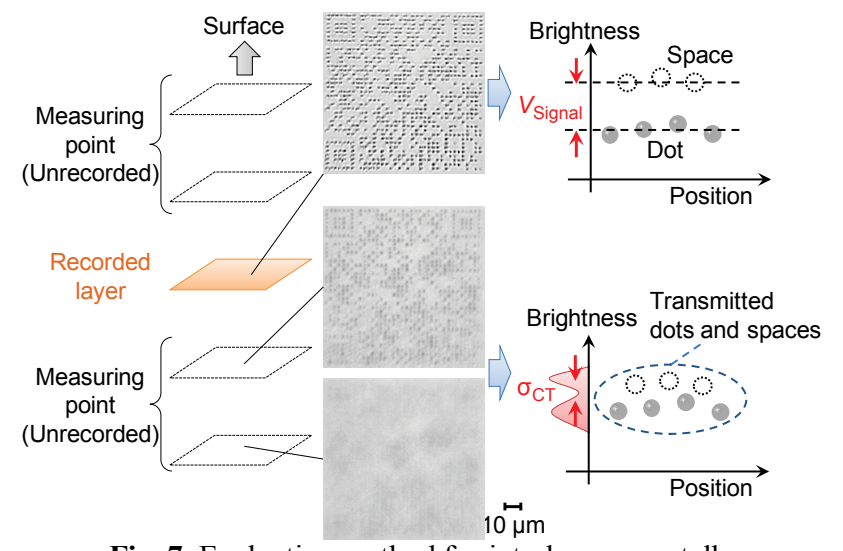

Fig. 7 Evaluation method for interlayer crosstalk.

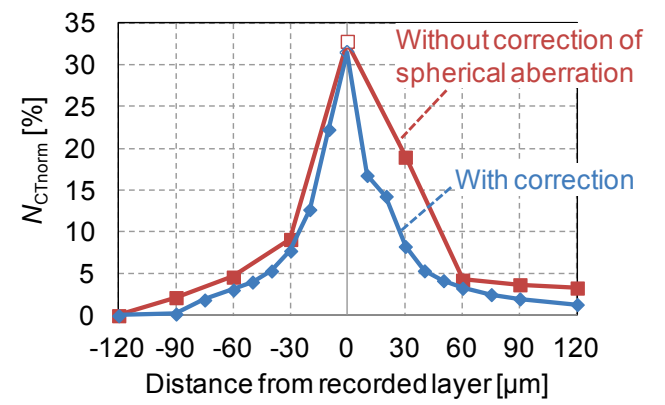

Fig. 8 Normalized interlayer-crosstalk noise in relation to distance from recorded layer.

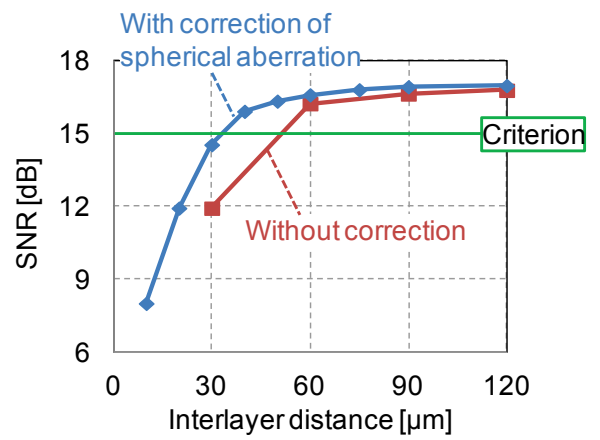

Fig. 9 SNR estimation for 26-layer sample.

\subsection{Recording and reading results of 26-layer sample}

Based on the derived recording condition, the 26-layer sample was made. Figure 10 shows a comparison of the microscopic image of a single layer sample and the 26layer sample. The QR codes ${ }^{\mathrm{TM}^{*}}$ shown in Fig. 11 were used as the recording pattern. The deepest and the shallowest layers of the 26-layer sample are called layer 0 and layer 25, respectively. Interlayer distance was set to $50 \mu \mathrm{m}$ to make best use of the correction range of spherical aberration of the objective lens $(50 \mu \mathrm{m} \times 26$ layers $=1.3 \mathrm{~mm})$. Spherical aberration was corrected in each layer and therefore recording energy could be kept constant. The recording density in each layer is $10.3 \mathrm{Mbyte} / \mathrm{in}^{2}{ }^{2}$, and thus the total recording density with 26 layers becomes 267 Mbyte/in. ${ }^{2}$, which is equivalent to that of a DVD (270 Mbyte/in. ${ }^{2}$ ). All dots of the sample shown in Fig. 10 were recorded successfully. However, interlayer crosstalk was observed as background noise in the 26-layer sample, especially on the center layer shown in Fig. 10(c).

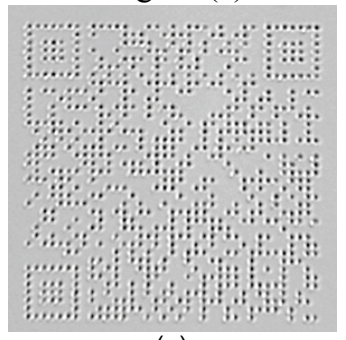

(a)

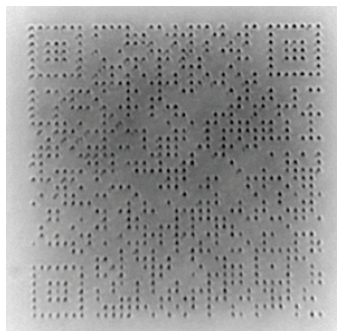

(c)

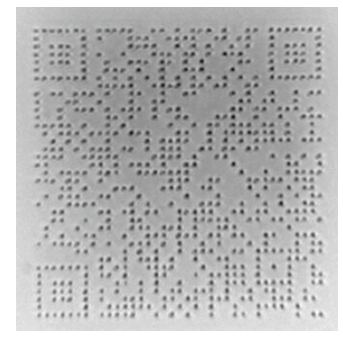

(b)

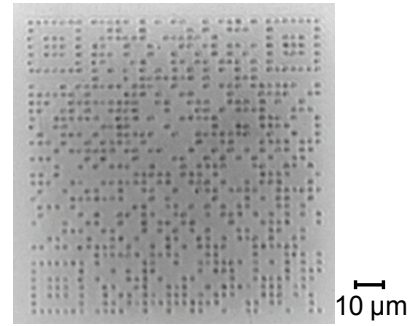

(d)
Fig. 10 Microscopic images of recorded sample. (a) Single layer sample. (b) Layer 0 of 26-layer sample. (c) Layer 12 of 26layer sample. (d) Layer 25 of 26-layer sample.

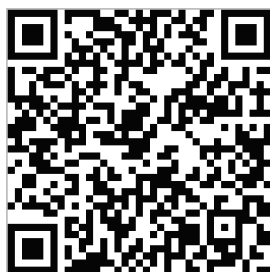

(a)

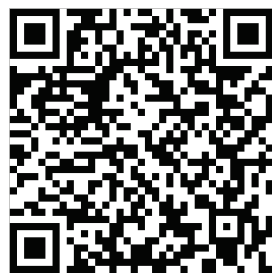

(b)
Fig. 11 Recording patterns. (a) Pattern for even-numbered layer. (b) Pattern for odd-numbered layer.

In order to improve reading quality, signal processing called unsharp masking was used. Its effect is shown in Fig. 12. It is a processing which subtracts a blurring image from an original image, resulting in removing low-frequency components and emphasizing dot edges. Figure 13 shows the reading result of the 26-layer sample. The SNR criterion of $15 \mathrm{~dB}$ or greater was achieved in all 26 layers, thus the data in $\mathrm{QR}$ cord $^{\mathrm{TM}}$ were correctly read using a $\mathrm{QR}$ cord $^{\mathrm{TM}}$ reader. 


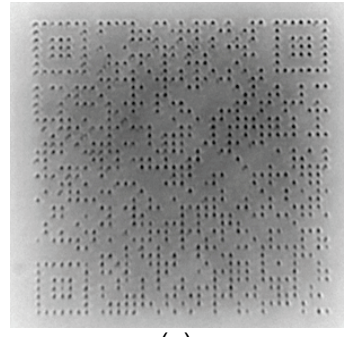

(a)

Fig. 12 Effect of unsharp masking. (a) Original image. (b) Image after unsharp masking.

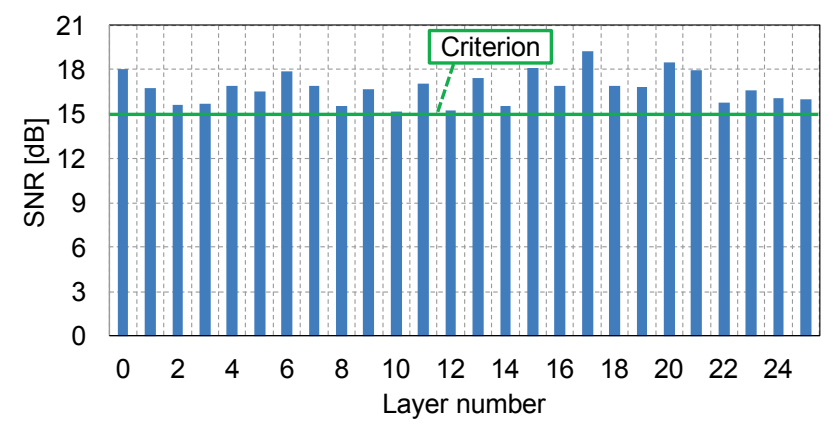

Fig. 13 Reading results of 26-layer sample.

\subsection{Evaluation of thermal resistance and lifetime esti- mation}

The thermal resistance of the recorded sample was evaluated and the lifetime of the sample was estimated by using the Arrhenius model. The results of the thermal resistance are shown in Fig. 14. Two samples were evaluated: the 26-layer sample (layer 12) and a sample made under a different recording condition (sample B) [6]. The SNR of sample B decreased with increasing the annealing time at $700{ }^{\circ} \mathrm{C}$ and $900{ }^{\circ} \mathrm{C}$. From these results, the lifetime at room temperature of sample B was estimated to be 319 million years [6]. In contrast, the 26-layer sample showed better thermal resistance and its SNR did not change after annealing at $1,000{ }^{\circ} \mathrm{C}$ for 120 minutes. This suggests that the lifetime of the 26-layer sample is over 319 million years.

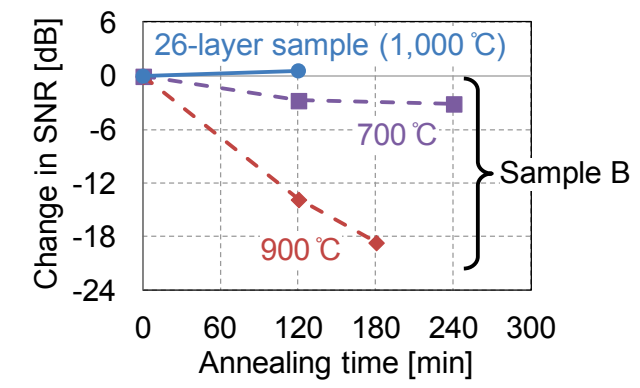

Fig. 14 Evaluation results of thermal resistance.

\section{Conclusion}

To increase the recording capacity in a storage system using fused silica, we studied the recording and reading conditions for a 26-layer sample with an interlayer of 50 $\mu \mathrm{m}$ made with a femtosecond laser and an SLM. The recording pulse energy and interlayer crosstalk were reduced by correcting spherical aberration. The recording quality was evaluated with an optical microscope. The SNR criterion of $15 \mathrm{~dB}$ was satisfied in all 26 layers by using unsharp masking. Thermal resistance at $1,000{ }^{\circ} \mathrm{C}$ for 120 minutes, which suggests a semi-permanent lifetime, was confirmed.

We reported 26-layer sample to realize the same recording capacity as DVD in this paper. The number of layer could be increased by using the objective lens with wider correction range of spherical aberration.

*QR $\operatorname{code}^{\mathrm{TM}}$ is a registered trademark of DENSO WAVE INCORPORATED.

\section{References}

[1] E. N. Glezer, M. Milosavljevic, L. Huang, R. J. Finlay, T.-H. Her, J. P. Callan and E. Mazur: Opt. Lett., 21, (1996) 2023. (Journals)

[2] E. N. Glezer and E. Mazur: Appl. Phys. Lett., 71, (1997) 882. (Journals)

[3] M. Watanabe, H. Sun, S. Juodkazis, T. Takahashi, S. Matsuo, Y. Suzuki, J. Nishii and H. Misawa: Jpn. J. Appl. Phys., 37, (1998) L1527. (Journals)

[4] J. Qiu, K. Miura and K. Hirao: Jpn. J. Appl. Phys., 37, (1998) 2263. (Journals)

[5] M. Watanabe, S. Juodkazis, H.-B. Sun, S. Matsuo, H. Misawa, M. Miwa and R. Kaneko: Appl. Phys. Lett., 74, (1999) 3957. (Journals)

[6] T. Watanabe, T. Shintani, K. Ono and T. Mine: IEICE Electron. Express, 6, (2009) 1569. (Journals)

[7] Y. Shimotsuma, P. G. Kazansky, J. Qiu and K. Hirao: Phys. Rev. Lett., 91, (2003) 247405. (Journals)

[8] Y. Shimotsuma, M. Sakakura, P. G. Kazansky, M. Beresna, J. Qiu, K. Miura and K. Hirao: Adv. Mater., 22, (2010) 4039. (Journals)

[9] M. Beresna, M. Gecevičius, P. G. Kazansky, T. Taylor, A. V. Kavokin: Appl. Phys. Lett., 101, (2012) 053120 (Journals)

[10] K. M. Davis, K. Miura, N. Sugimoto and K. Hirao: Opt. Lett., 21, (1996) 1729. (Journals)

[11] K. Miura, J. Qiu, H. Inouye, T. Mitsuyu and K. Hirao: Appl. Phys. Lett., 71, (1997) 3329. (Journals)

[12] M. Sakakura, T. Sawano, Y. Shimotsuma, K. Miura and K. Hirao: Jpn. J. Appl. Phys., 48, (2009) 126507. (Journals)

[13] C. Mauclair, G. Cheng, N. Huot, E. Audouard, A. Rosenfeld, I. V. Hertel and R. Stoian: Opt. Express, 17, (2009) 3531. (Journals)

[14] M. Pospiech, M. Emons, A. Steinmann, G. Palmer, R. Osellame, N. Bellini, G. Cerullo and U. Morgner: Opt. Express, 17, (2009) 3555. (Journals)

[15] M. Sakakura, T. Sawano, Y. Shimotsuma, K. Miura and K. Hirao: Opt. Lett., 36, (2011) 1065. (Journals)

[16] M. Shiozawa, T. Watanabe, E. Tatsu, M. Umeda, T. Mine, Y. Shimotsuma, M. Sakakura, M. Nakabayashi, K. Miura and K. Watanabe: Proc. International Symposium on Optical Memory, (2012) p. 26. (Conference Proceedings)

[17] M. Shiozawa, T. Watanabe, E. Tatsu, M. Umeda, T. Mine, Y. Shimotsuma, M. Sakakura, M. Nakabayashi, K. Miura and K. Watanabe: Proc. International Symposium on Optical Memory, (2012) p. 28. (Conference Proceedings)

[18]H. Mikami, K. Osawa, E. Tatsu and K. Watanabe: Jpn. J. Appl. Phys., 51, (2012) 08JD01. (Journals)

(Received: August 1, 2013, Accepted: December 13, 2013) 